\title{
ROBUST SIGNAL RECOVERY FROM INCOMPLETE OBSERVATIONS
}

\author{
Emmanuel Candès and Justin Romberg \\ Applied and Computational Mathematics, Caltech \\ Pasadena, CA 91125
}

\begin{abstract}
Recently, a series of exciting results have shown that it is possible to reconstruct a sparse signal exactly from a very limited number of linear measurements by solving a convex optimization program. If our underlying signal $f$ can be written as a superposition of $B$ elements from a known basis, it is possible to recover f from a projection onto a generic subspace of dimension about $B \log N$. Moreover, the procedure is robust to measurement error; adding a perturbation of size $\epsilon$ to the measurements will not induce a recovery error of more than a small constant times $\epsilon$.

In this paper, we will briefly overview these results, and show how the recovery via convex optimization can be implemented in an efficient manner, and present some numerical results illustrating the practicality of the procedure.
\end{abstract}

\section{INTRODUCTION}

\subsection{Signal recovery from incomplete measurements}

A standard imaging problem is to recover a finite signal $x_{0} \in \mathbb{R}^{N}$ from a series of indirect measurements:

$$
y_{k}=\left\langle x_{0}, \phi_{k}\right\rangle, \quad k=1, \ldots, K .
$$

The measurements $y$ are linear in that $y=\Phi x_{0}$, where $\Phi$ is the $K \times N$ matrix formed by taking the $\phi_{k}$ as rows. We will be particularly interested in the case where $K \ll N$, and we are faced with a severely under-determined inverse problem. A series of recent results [1-5] have shown that if $x_{0}$ is sparse in that it has very few significant components, it can be reconstructed exactly by solving the convex optimization program

$$
\left(P_{1}\right) \quad \min \|x\|_{\ell_{1}} \quad \text { such that } \quad \Phi x=y .
$$

To make the conditions on when $x_{0}$ can be recovered perfectly precise, let $T \subset\{1, \ldots, N\}$ be a subset of indices, and let $\Phi_{T}$ be the submatrix formed by extracting the columns of $\Phi$ corresponding to the indices in $T$. We say that $\Phi_{T}$ is a restricted isometry for sets of size $S$ if there exists a $\delta_{S}<1$ such that

$$
\left(1-\delta_{S}\right) \cdot\|c\|_{\ell_{2}} \leq\left\|\Phi_{T} c\right\|_{\ell_{2}} \leq\left(1+\delta_{S}\right) \cdot\|c\|_{\ell_{2}}
$$

for all coefficient sequences $c$ supported on $T$, and all sets $T$ with fewer than $S$ elements: $|T| \leq S$. Say we are given measurements $y$, and call $\hat{x}$ the solution to $\left(P_{1}\right)$ above. If for a given $S$, the restricted isometry constants obey the technical condition [4]

$$
3 \delta_{4 S}+\delta_{3 S}<2
$$

Email: jrom@acm.caltech.edu, Phone: (626) 395-5760. the recovery error can be bounded by

$$
\left\|\hat{x}-x_{0}\right\|_{\ell_{2}} \leq C \cdot S^{-1 / 2} \cdot\left\|x_{0, S}-x_{0}\right\|_{\ell_{1}},
$$

where $x_{0, S}$ is the best $S$-term approximation of $x_{0}$ formed by taking the $S$ largest values of $x_{0}$ and setting the rest to zero ${ }^{1}$. If $x_{0}$ has $S$ or fewer nonzero components, the error in (3) is zero, and the recovery is perfect.

The recovery result (3) is also meaningful when $x_{0}$ is compressible, rather than perfectly sparse. Suppose that $x_{0}$ obeys the power law

$$
\left|x_{0}\right|_{(n)} \leq C \cdot n^{-1 / p}
$$

for some $p<1$, where $\left|x_{0}\right|_{(n)}$ is the $n$th largest component (by magnitude) of $x_{0}$. The primary feature of such vectors is that their $S$-term approximations are guaranteed to obey a similar power law:

$$
\left\|x_{0}-x_{0, S}\right\|_{\ell_{2}} \leq C \cdot S^{-\alpha}
$$

where $\alpha=1 / p-1 / 2$, and $x_{0, S}$ is the vector formed by copying the $S$ largest components of $x_{0}$ and setting the rest to zero. While compressible $x_{0}$ are not exactly sparse, they can be closely approximated by vectors which are. For $x_{0}$ as in (4), the right hand side of (3) can also be bounded by

$$
S^{-1 / 2} \cdot\left\|x_{0, S}-x_{0}\right\|_{\ell_{1}} \leq C \cdot S^{-\alpha} \text {. }
$$

Thus the recovery error from partial information is on the same order as the optimal $S$-term approximation.

Measurement ensembles which obey (2) for $K$ not too much larger than $S$ are easily constructed. If we choose each entry of $\Phi$ as a Gaussian random variable with variance $1 / K,(2)$ is obeyed for $[2,3]$

$$
S \leq C \cdot \frac{K}{\log (N / K)}
$$

with overwhelming probability (see also [5] for a different formulation). We will refer to such a $\Phi$ as a Gaussian measurement ensemble. Another example is the partial Fourier ensemble, created by taking $K$ rows at random from the discrete Fourier transform matrix, and renormalizing the columns. With overwhelming probability, the restricted isometry constants of the partial Fourier ensemble obey (2) for

$$
S \leq C \cdot \frac{K}{\log ^{6}(N / K)} .
$$

Combining equations (3) and (6) shows us how powerful the reconstruction procedure is. First, let us envision a kind oracle procedure for measuring a sparse $x_{0}$. Suppose that we know which

\footnotetext{
${ }^{1}$ Throughout the entirety of this paper, we will forgo explicit calculation of constants which do not depend on the quantities of interest. These constants will be denoted everywhere as $C$.
} 
components of $x_{0}$ are the most important (i.e. have the greatest magnitude). If we form $x_{0, K}$ by sampling $x_{0}$ at the $K$ "largest" locations (and setting the rest to zero), the reconstruction error quickly goes to zero as $K$ increases:

$$
\left\|x_{0}-x_{0, K}\right\|_{\ell_{2}} \leq C \cdot K^{-\alpha}
$$

where again $\alpha=1 / p-1 / 2$, and the constant depends only on $p$. Now suppose (more realistically, most would say), that we have no such oracle information. Instead of taking $K$ samples of $x_{0}$, we take $K$ measurements again Gaussian test functions. Since (2) will be obeyed for $S$ as in (6), equation (5) tells us that

$$
\left\|\hat{x}-x_{0}\right\|_{\ell_{2}} \leq C \cdot\left(\frac{K}{\log (N / K)}\right)^{-\alpha}
$$

Thus the recovery error decreases to zero at nearly the same rate as the oracle nonlinear approximation error, even though the measurements are completely nonadaptive. The number of measurements we need to take depend only on the inherent complexity of the signal.

The recovery procedure (solving $\left(P_{1}\right)$ ) is very nonlinear. In spite of this, it can be made amazingly stable to the corruption of the measurements $y$. Suppose now that we observe $y=\Phi x_{0}+e$, where $e$ is an unknown perturbation whose size can be bounded $\|e\|_{\ell_{2}} \leq \epsilon$. In place of $\left(P_{1}\right)$, we solve the relaxed problem

$$
\left(P_{2}\right) \quad \min \|x\|_{\ell_{1}} \text { such that }\|\Phi x-y\|_{\ell_{2}} \leq \epsilon .
$$

Call $\hat{x}$ the solution to $\left(P_{2}\right)$. In [4], it was shown that if the restricted isometry constants for sets of size $S$ again obey (2), then the recovery error can be bounded by

$$
\left\|\hat{x}-x_{0}\right\|_{\ell_{2}} \leq C \cdot\left(\epsilon+S^{-1 / 2} \cdot\left\|x_{0, S}-x_{0}\right\|_{\ell_{1}}\right)
$$

where $C$ is a small constant $(C<10$ in many interesting cases). Thus the error in the recovery is on the same order as the larger of the approximation error and the size of the measurement error.

Finally, the methodology extends beyond the traditional sense of sparsity. It is popular in the field of image restoration to model an image $x_{0}$ as having a small total variation norm,

$$
\|x\|_{T V}=\sum_{i j} \sqrt{\left(D_{i j}^{h} x\right)^{2}+\left(D_{i j}^{v} x\right)^{2}}
$$

where $D_{i j}^{h}$ and $D_{i j}^{v}$ are the local difference operators the pixel in the $i$ th row and $j$ th column in the $n \times n$ digital image (now $N=n^{2}$ is the total number of pixels):

$$
\begin{aligned}
& D_{i j}^{h} x= \begin{cases}x(i+1, j)-x(i, j) & 0 \leq i \leq n-1 \\
0 & i=n\end{cases} \\
& D_{i j}^{v} x= \begin{cases}x(i, j+1)-x(i, j) & 0 \leq j \leq n-1 \\
0 & j=n\end{cases}
\end{aligned}
$$

Thus $\|x\|_{T V}$ is roughly the $\ell_{1}$ norm of the discretized gradient of the image at each pixel, and recovery via

$$
(T V) \min _{x}\|x\|_{T V} \quad \text { such that }\|\Phi x-y\|_{\ell_{2}} \leq \epsilon
$$

produces the image with the sparsest gradient that explains the measurements we have taken. From this standpoint, the theory for solving $(T V)$ parallels that for the standard $\ell_{1}$ problem.

The next section shows how problems $\left(P_{2}\right)$ and $(T V)$ can be reformulated as second-order cone programs, and hence solve using standard software. Section 3 presents some numerical results for recovery via $\left(P_{2}\right)$ and $(T V)$.

\section{SECOND-ORDER CONE PROGRAMMING}

It has been noted many times in the literature (most explicitly in [6]) that the problem $\left(P_{1}\right)$, also known as Basis Pursuit, can be recast as a linear program (LP). The great advances in interior point methods for linear programs made over the past decade (see [7,8]) thus allow us to solve $\left(P_{1}\right)$ for fairly large problem sizes.

Although they are not linear pograms, our relaxed problems $\left(P_{2}\right)$ and $(T V)$ can be recast as second-order cone programs (SOCP). A SOCP is a program of the form [7]

$$
\min c_{0}^{T} z \quad \text { s.t. }\left\|A_{i} z-b_{i}\right\|_{\ell_{2}} \leq c_{i}^{T} z+d_{i}, \quad i=1, \ldots, P .
$$

Progress in solving SOCPs has virtually matched that of linear programming; interior point methods $[7,9]$ exist whose performance (in theory and in practice) is more or less the same as their LP counterparts.

It is clear how the relaxed $\ell_{1}$ problem $\left(P_{2}\right)$ falls into the SOCP framework. In lieu of $\left(P_{2}\right)$, we simply solve the equivalent problem

$$
\left(P_{2}^{\prime}\right) \min _{x, u} \sum_{i=1}^{N} u_{i} \quad \text { such that } \begin{aligned}
& \left|x_{j}\right| \leq u_{j}, j=1, \ldots, N \\
& \\
& \|\Phi x-y\|_{\ell_{2}} \leq \epsilon
\end{aligned}
$$

which is a $2 N$ dimensional problem with $N+1$ conic constraints.

With a little work, the program $(T V)$ can also be recast as an SOCP (see $[10,11]$ for a complete discussion). From (9) above, we can write

$$
\|x\|_{T V}=\sum_{i j}\left\|D_{i j} x\right\|_{\ell_{2}}
$$

where each $D_{i j}$ is a $2 \times N$ matrix

$$
D_{i j} x=\left[\begin{array}{c}
D_{i j}^{h} x \\
D_{i j}^{v} x
\end{array}\right] .
$$

Then an SOCP which is equivalent to $(T V)$ is

$$
\begin{aligned}
\left(T V^{\prime}\right) \min _{x, u} \sum_{i j} u_{i j} \text { such that } \begin{array}{l}
\left\|D_{i j} x\right\|_{\ell_{2}} \leq t_{i j} \\
\\
\end{array} \mid \Phi x-y \|_{\ell_{2}} \leq \epsilon
\end{aligned}
$$

which is again a program in $2 N$ variables with $N+1$ constraints (again, the index $i j$ runs over each of the $N:=n^{2}$ pixels in the image).

The problems $\left(P_{2}^{\prime}\right),\left(T V^{\prime}\right)$ can be solved using standard $\log$ barrier techniques. We adapt the generic technique presented in [7, Chap. 11]. Let $z:=(x, u)$ be the $2 N$ dimensional search vector and $f_{i}(z):=\left\|A_{i} z-b_{i}\right\|_{\ell_{2}}^{2}-\left(c_{i}^{T} z+d_{i}\right)^{2}, \quad i=1, \ldots, P$ be the set of $N+1$ inequality constraints. The SOCP is transformed into a series of unconstrained problems parameterized by $\tau>0$ :

$$
\min _{z} c_{0}^{T} z+\frac{1}{\tau} \sum_{i=1}^{P}-\log \left(-f_{i}(z)\right)
$$

where the first $N$ entries of $c_{0}$ are zero, and the second $N$ are one: $c_{0}=\left(\begin{array}{ll}0 & 1\end{array}\right)^{T}$. The inequality constraints have been incorporated into the functional via a penalty function ${ }^{2}$ which is infinite when the constraint is violated (or even met exactly), and smooth elsewhere. As $\tau \rightarrow \infty$, the solution of (11) approaches the solution of (10); in

\footnotetext{
${ }^{2}$ The choice of $-\log (-x)$ for the barrier function is not arbitrary, it has a property (termed self-concordance) that is very important for quick convergence of (11) to (10) both in theory and in practice (see the very nice exposition in [12]).
} 
fact, the solution of $(11)$ is within $(N+1) / \tau$ of the optimal value of (10).

The subproblem (11) is solved for a series of values $\tau^{r}, r=$ $1, \ldots R$ with $\tau^{r+1}=\mu \tau^{r}$ for some $\mu>1$, and $\tau^{R}$ is chosen so that $(N+1) / \tau^{R}$ is acceptably small. The idea is that each of these smooth subproblems can be solved to fairly high accuracy with just a few iterations of Newtons method, especially since we can use the solution at iteration $r$ as the starting point for iteration $r+1$.

At log-barrier iteration $r$, Newton's method (which is again iterative) proceeds by forming a series of quadratic approximations to (11), and minimizing each by solving a system of equations. Let $f_{0}(z)$ denote the functional being minimized in (11). The quadratic approximation of $f_{0}$ around a point $z$ is given by

$$
f_{0}(z+\delta z) \approx z+\left\langle g_{z}, \delta z\right\rangle+\frac{1}{2}\left\langle H_{z} \delta z, \delta z\right\rangle:=q(z+\delta z),
$$

where $g_{z}$ is the gradient

$$
g_{z}=c_{0}+\frac{1}{\tau} \sum_{i} \frac{1}{-f_{i}(z)} \nabla f_{i}(z)
$$

and $H_{z}$ is the Hessian matrix

$H_{z}=\frac{1}{\tau} \sum_{i} \frac{1}{f_{i}(z)^{2}} \nabla f_{i}(z)\left(\nabla f_{i}(z)\right)^{T}+\frac{1}{\tau} \sum_{i} \frac{1}{-f_{i}(z)} \nabla^{2} f_{i}(z)$.

The $\delta z$ that minimizes $q(z+\delta z)$ is the solution to the set of linear equations

$$
\tau H_{z}=-\tau g_{z} .
$$

We see that at their core, interior point methods for solving $\left(P_{2}^{\prime}\right),\left(T V^{\prime}\right)$ involve taking a series of Newton steps whose direction is chosen by solving a $K \times K$ system of equations. At first, as $K$ could be quite large (on the order of $10^{5}$ in the experiments below, for example), this task seems quite daunting. However, if $\Phi$ and its adjoint (and thus $H_{z}$ and its adjoint) can be applied quickly (such as when $\Phi$ is a partial Fourier ensemble), then this system itself can be solved using conjugate gradients [13] or other similar algorithms.

With $\delta z$ in hand, we have the Newton step direction. The step length $s \leq 1$ is chosen so that:

1. $f_{i}(z+s \delta z)<0$ for all $i=1, \ldots, m$. We require the algorithm to stay in the interior of the set defined by the inequality constraints.

2. The functional has decreased suffiently:

$$
f_{0}(z+s \delta z)<f_{0}(z)+\alpha s \delta z\left\langle g_{z}, \delta z\right\rangle,
$$

where $\alpha$ is a user-specified parameter (each of the implementations below uses $\alpha=0.01$ ). This requirement basically states that the decrease must be within a certain percentage of that predicted by the linear model at $z$.

To find such an $s$, we can use a simple backtracking line search. Starting with $s=1$, we decrease $s$ by a factor of $1 / 2$ until both conditions above are satisfied.

The complete log-barrier implementation for each problem follows the outline:

1. Inputs: a feasible starting point $z^{0}$, a tolerance $\eta$, and parameters $\mu$ and an initial $\tau^{1}$. Set $r=1$.

2. Solve (11) via Newton's method (followed by the backtracking line search), using $z^{r-1}$ as an initial point. Call the solution $z^{r}$.
3. If $(N+1) / \tau^{r}<\eta$, terminate and return $z^{r}$.
4. Else, set $\tau^{r+1}=\mu \tau^{r}, r=r+1$ and go to step 2 .

In fact, we can calculate in advance how many iterations the logbarrier algorithm will need:

$$
\text { barrier iterations }=\left\lceil\frac{\log (N+1)-\log \eta-\log \tau^{1}}{\log \mu}\right\rceil .
$$

The final issue is the selection of $\tau^{1}$. In our experience, a conservative choice usually suffices; it can be set of that the accuracy $(N+1) / \tau^{1}$ after the first iteration is equal to the value of the initial functional $c_{0}^{T} z^{0}$.

The authors have recently released a code package [14] that includes an implementation of this algorithm.

\section{EXAMPLES}

In this section, we demonstrate the effectiveness of $(T V)$ in recovering photograph-like images from partial measurements with some numerical experiments. We will examine the case where the measurements are not only incomplete, but are also inaccurate by subjecting $y$ to two types of corruption: additive white Gaussian noise (AWGN) and quantization error.

Our measurement ensemble $\Phi$ in these experiments will be the scrambled Fourier ensemble, formed by taking a partial Fourier ensemble and randomly permuting the columns. In practice, this ensemble seems to perform as well as a "pure" Gaussian ensemble, but has the advantage that $\Phi$ (and its adjoint) can be applied efficiently, making our recovery procedure computationally feasible for images with more than a few thousand pixels.

We measured each of the 4 well-known $256 \times 256$ pixel images listed in Table 1 with a $25000 \times 65536$ scrambled Fourier ensemble. Recovery results (obtained by solving $(T V)$ with a small value of $\epsilon)$ for noise-free measurements are shown in Table 1; as a reference, the size of the Daubechies- 8 wavelet nonlinear approximation required to get the same error is also tabulated. From about 4 times as many nonadaptive measurements, we are able to match the optimal wavelet approximation.

The recovery is well-behave when the measurements are corrupted by AWGN: $y_{k}=\left\langle x_{0}, \phi_{k}\right\rangle+e_{k}$, where $e_{k} \sim \mathcal{N}\left(0, \sigma^{2}\right)$ for a given value of $\sigma$. The size of the perturbation $\|e\|_{\ell_{2}}^{2}$ is a chi-square random variable with mean $\sigma^{2} K$ and standard deviation $\sigma^{2} \sqrt{2 K}$. The probability that $\|e\|_{\ell_{2}}^{2}$ exceeds its mean plus two standard deviations is small, as such we will recover the images by solving (TV) with

$$
\epsilon^{2}=\sigma^{2}(K+2 \sqrt{2} K)
$$

Recovery results for $\sigma=5 \cdot 10^{-4}$ (for a signal-to-noise-ratio of $\left\|\Phi x_{0}\right\|_{\ell_{2}} /\|e\|_{\ell_{2}} \approx 4.5$ ) are charted in Table 2 . In all cases, the recovery error is about the size of $\epsilon$, indicating that in practice, the constants in (8) are not too large.

The recovery is also stable in practice when the perturbation is signal dependent. Instead of adding AWGN, we quantize the $y_{k}$ to the closest of 10 pre-defined, equally spaced levels, i.e. we limit the resolution of the measurements to one digit. To choose $\epsilon$, we use a crude (but effective) model for the quantization error. If the $e_{k}$ were independent uniform random variables over the interval $[-q / 2, q / 2],\|e\|_{\ell_{2}}^{2}$ would have mean $K q^{2} / 12$ and standard deviation $\sqrt{K} q^{2} /(6 \sqrt{5})$. Again, we would expect $\|e\|_{\ell_{2}}^{2}$ to be no larger than its mean plus two standard deviations, so we will use

$$
\epsilon^{2}=K q^{2} / 12+\sqrt{K} q^{2} /(3 \sqrt{5}) .
$$


Table 1. Noiseless image recovery results from solving (TV) with 25000 measurements (less than half the data). The bottom row is the number of terms in a Daubechies- 8 wavelet approximation needed to match the recovery error.

\begin{tabular}{|c||c|c|c|c|}
\hline & Lenna & Cameraman & Boats & Fruit \\
\hline \hline$\left\|\hat{x}_{T V}-x_{0}\right\| \ell_{2}$ & 0.0453 & 0.0400 & 0.0392 & 0.0262 \\
\hline$K_{\text {wavelet }}$ & 6557 & 8121 & 6857 & 6065 \\
\hline
\end{tabular}

Table 2. Image recovery results. 25000 Measurements of the Lenna, Cameraman, Boats and Fruit images were corrupted by adding white noise with $\sigma=5 \cdot 10^{-4}$. The image was recovered in by solving $(T V)$ with $\epsilon=0.08$. An example is shown in Figure 1(c).

\begin{tabular}{|c||c|c|c|c|}
\hline & Lenna & Cameraman & Boats & Fruit \\
\hline \hline$\left\|\hat{x}_{T V}-x_{0}\right\|_{\ell_{2}}$ & 0.0866 & 0.0941 & 0.0837 & 0.0665 \\
\hline
\end{tabular}

The results of the recovery from quantized measurements are shown in Table 3 . Note that the recovery errors are about 1.5 times as large as the corruption error. Also note that despite the crudeness of the model used to predict the size of the error, the value (14) chosen for $\epsilon$ is very close to the size of the actual error.

\section{REFERENCES}

[1] E. Candès, J. Romberg, and T. Tao, "Robust uncertainty principles: Exact signal reconstruction from highly incomplete frequency information," to appear in IEEE Trans. Inform. Theory, February 2006, Available on theArXiV preprint server: math. GM/ 0409186.

[2] E. Candès and T. Tao, "Near-optimal signal recovery from random projections and universal encoding strategies," submitted to IEEE Trans. Inform. Theory, November 2004, Available on the ArXiV preprint server: math.CA/0410542.

[3] E. J. Candès and T. Tao, "Decoding by linear programming," IEEE Trans. Inform. Theory, December 2005.

[4] E. Candès, J. Romberg, and T. Tao, "Stable signal recovery from incomplete and inaccurate measurements," to appear in Communications on Pure and Applied Mathematics, 2006.

[5] D. L. Donoho, "Compressed sensing," submitted to IEEE Trans. Inform. Theory, September 2004.

[6] S. S. Chen, D. L. Donoho, and M. A. Saunders, "Atomic decomposition by basis pursuit," SIAM J. Sci. Comput., vol. 20, pp. 33-61, 1999.

Table 3. Recovery from quantized measurements. 25000 Measurements of the Lenna, Cameraman, Boats and Fruit images were quantized to 1 digit, (10 bins). Scalar, uniform quantization was used between the maximum and minimum of the measurements. The images were recovered using (TV) with the values of $\epsilon$ tabulated below. An example is shown in Figure 1(d).

\begin{tabular}{|c||c|c|c|c|}
\hline & Lenna & Cameraman & Boats & Fruit \\
\hline \hline$\left\|\hat{x}_{T V}-x_{0}\right\|_{\ell_{2}}$ & 0.1136 & 0.1090 & 0.0843 & 0.0773 \\
\hline$\epsilon$ (using (14)) & 0.0751 & 0.0685 & 0.0511 & 0.0682 \\
\hline actual $\|e\|_{\ell_{2}}$ & 0.0743 & 0.0677 & 0.0509 & 0.0679 \\
\hline
\end{tabular}

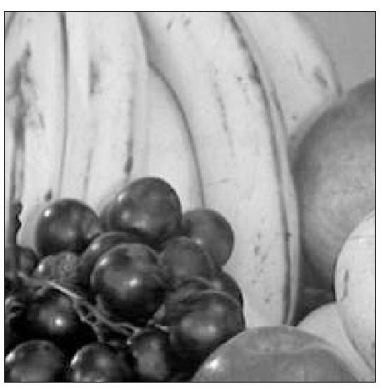

(a)

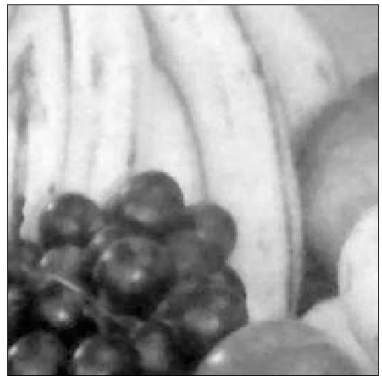

(c)

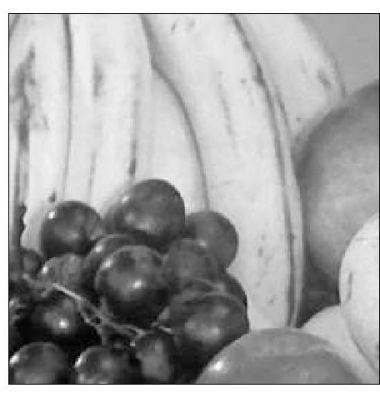

(b)

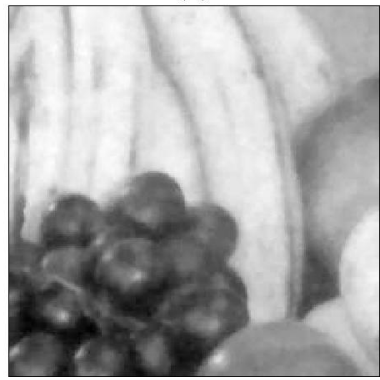

(d)
Fig. 1. Recovery example for the noisy 'Fruits' image. (a) Original image. (b) Recovered from 25000 noiseless measurements (see Table 1) via (TV). (c) Recovered from 25000 measurements corrupted with additive white Gaussian noise (see Table 2) via (TV). (d) Recovered from 25000 measurements quantized to one digit (see Table 3).

[7] S. Boyd and L. Vandenberghe, Convex Optimization, Cambridge University Press, 2004.

[8] S. J. Wright, Primal-Dual Interior-Point Methods, SIAM Publications, 1997.

[9] F. Alizadeh and D. Goldfarb, "Second-order cone programming," Math. Program., Ser B, vol. 95, pp. 3-51, 2003.

[10] T. Chan, G. Golub, and P. Mulet, "A nonlinear primal-dual method for total variation-based image restoration," SIAM J. Sci. Comput., vol. 20, pp. 1964-1977, 1999.

[11] D. Goldfarb and W. Yin, "Second-order cone programming methods for total variation-based image restoration," Tech. Rep., Columbia University, 2004.

[12] J. Renegar, A mathematical view of interior-point methods in convex optimization, MPS-SIAM Series on Optimization. SIAM, 2001.

[13] J. R. Shewchuk, "An introduction to the conjugate gradient method without the agonizing pain," Manuscript, August 1994.

[14] " $\ell_{1}$-MAGIC," http://www.11-magic.org. 